\title{
Usefulness of a Simple Clinical Risk Prediction Method, Modified ACEF Score, for Transcatheter Aortic Valve Implantation
}

Takahide Arai, MD; Thierry Lefèvre, MD; Kentaro Hayashida, MD, PhD; Yusuke Watanabe, MD; Stephen A. O'Connor, MD; Thomas Hovasse, MD; Mauro Romano, MD; Philippe Garot, MD; Erik Bouvier, MD; Bernard Chevalier, MD; Marie-Claude Morice, MD

Background: We assessed the predictive accuracy of a simple risk score, modified age, creatinine clearance, ejection fraction (ACEFmodif) score, for outcome of transcatheter aortic valve implantation (TAVI).

Methods and Results: We prospectively included 703 consecutive patients undergoing TAVI. Patients were divided into low, middle and high ACEFmodif tertiles. Increased ACEFmodif score was associated with a significantly higher 1 -year mortality rate $(22 \%, 28 \%$ and $36 \%, \mathrm{P}<0.01)$ and higher risk of acute kidney injury $(\mathrm{AKI} ; 10 \%, 10 \%$ and $22 \%$, $\mathrm{P}<0.01)$. On multivariate logistic regression analysis, ACEFmodif score was the only independent predictor of AKI. On multivariate Cox regression, ACEFmodif score was an independent predictor of 1-year cumulative mortality. Although the area under curve (AUC) showed that all risk scores poorly predicted the incidence of AKI and 1-year cumulative mortality, ACEFmodif score was more efficient in predicting the incidence of AKI compared with STS, LES and ES II (AUC, 0.61, 0.55, 0.54, 0.57, respectively). Furthermore, ACEFmodif score had similar accuracy in predicting 1-year mortality compared with other risk scores (AUC, 0.61, 0.61, 0.61, 0.61, respectively).

Conclusions: ACEFmodif score may provide useful information for predicting AKI, 30-day and 1-year mortality in patients undergoing TAVI, but these results need further confirmation. (Circ J 2015; 79: 1496-1503)

Key Words: Acute kidney injury; Modified age, creatinine clearance, ejection fraction score; Severe symptomatic aortic stenosis; Transcatheter aortic valve implantation

$\mathbf{T}$ ranscatheter aortic valve implantation (TAVI) is being increasingly used as a treatment option for patients with severe symptomatic aortic stenosis (AS) who are considered at high risk for surgical aortic valve replacement (SAVR). ${ }^{1-4}$ Despite its minimally invasive nature, TAVI is invariably associated with complications that may affect outcomes. Consequently, pre-procedure evaluation of risks should be carried out thoroughly. Although risk assessment scores such as the Logistic European System for Cardiac Operative Risk Evaluation (LES), the European System for Cardiac Operative Risk Evaluation Score II (ES II) and the Society of Thoracic Surgeons (STS) predicted risk mortality score have proved useful, 5,6 their predictive value remains inadequate despite a relatively high number of variables.

Age, creatinine clearance, ejection fraction (ACEF) score is a novel and simple scoring method that includes only 3 variables. ${ }^{7}$ This simple scoring system has been tested in patients undergoing coronary artery bypass grafting (CABG) and percutaneous coronary intervention (PCI) and has been shown to be helpful in predicting outcome ${ }^{8,9}$ The modified ACEF (ACEFmodif) score includes creatinine clearance as a semicontinuous variable instead of serum creatinine and has been reported to increase the accuracy of the original ACEF score among PCI patients. ${ }^{10-12}$ The usefulness of this score in patients undergoing TAVI, however, has not been previously assessed in a large cohort of patients. The aim of this study was, therefore, to assess the accuracy of the ACEFmodif score in predicting the outcome of patients undergoing TAVI.

\section{Methods}

\section{Study Design}

From October 2006, all consecutive high-risk patients with severe symptomatic AS treated with TAVI were prospectively

Received November 13, 2014; revised manuscript received February 19, 2015; accepted March 15, 2015; released online May 1, 2015 Time for primary review: 24 days

Department of Interventional Cardiology (T.A., T.L., K.H., Y.W., S.A.O., T.H., P.G., E.B., B.C., M.-C.M.), Department of Cardiovascular Surgery (M.R.), Institut Cardiovasculaire Paris Sud, Massy, France; Department of Cardiology, Keio University School of Medicine, Tokyo (K.H.); and Department of Cardiology, Teikyo University School of Medicine, Tokyo (Y.W.), Japan

Mailing address: Thierry Lefèvre, MD, FESC, FSCAI, Department of Interventional Cardiology, Hôpital Privé Jacques Cartier, Générale de Santé, 6 avenue du Noyer Lambert, FR-91300 Massy, France. E-mail: t.lefevre@angio-icps.com

ISSN-1346-9843 doi:10.1253/circj.CJ-14-1242

All rights are reserved to the Japanese Circulation Society. For permissions, please e-mail: cj@j-circ.or.jp 


\begin{tabular}{|c|c|c|c|c|}
\hline & $\begin{array}{l}\text { Low ACEF modif } \\
(0.6-2.1)(n=236)\end{array}$ & $\begin{array}{l}\text { Middle ACEF modif } \\
(2.2-3.8)(n=237)\end{array}$ & $\begin{array}{l}\text { High ACEF modif } \\
(3.9-9.6)(n=230)\end{array}$ & P-value \\
\hline \multicolumn{5}{|l|}{ Baseline characteristics } \\
\hline Age (years) & $80.7 \pm 7.6$ & $84.1 \pm 5.0$ & $85.2 \pm 5.7$ & $<0.01$ \\
\hline Male & $106(45 \%)$ & $116(49 \%)$ & $136(59 \%)$ & $<0.01$ \\
\hline $\operatorname{BMI}\left(\mathrm{kg} / \mathrm{m}^{2}\right)$ & $27.7 \pm 5.4$ & $25.4 \pm 4.0$ & $24.3 \pm 3.3$ & $<0.01$ \\
\hline $\mathrm{BSA}\left(\mathrm{m}^{2}\right)$ & $1.79 \pm 0.22$ & $1.73 \pm 0.19$ & $1.70 \pm 0.18$ & $<0.01$ \\
\hline NYHA classification (III/IV) & $190(81)$ & $204(86)$ & $196(85)$ & 0.27 \\
\hline Peripheral artery disease & 78 (33) & $66(28)$ & 78 (34) & 0.34 \\
\hline Prior $\mathrm{Ml}$ & $18(8)$ & $21(9)$ & $29(13)$ & 0.18 \\
\hline Prior $\mathrm{PCl}$ & $47(20)$ & $56(24)$ & $75(33)$ & $<0.01$ \\
\hline Prior CAGB & $35(15)$ & $26(11)$ & $32(14)$ & 0.35 \\
\hline Prior cardiac surgery & $40(17)$ & $30(13)$ & $32(14)$ & 0.42 \\
\hline Prior stroke & $26(11)$ & $12(5)$ & $23(10)$ & 0.07 \\
\hline Atrial fibrillation & $70(30)$ & $66(28)$ & $80(35)$ & 0.27 \\
\hline Diabetes mellitus & $59(25)$ & $54(23)$ & $50(22)$ & 0.68 \\
\hline Hypertension & $169(73)$ & $151(64)$ & $156(68)$ & 0.09 \\
\hline Dyslipidemia & $121(52)$ & $120(51)$ & $108(47)$ & 0.52 \\
\hline Cancer & $32(14)$ & $28(12)$ & $32(14)$ & 0.75 \\
\hline COPD & $190(81)$ & $204(86)$ & $196(85)$ & 0.77 \\
\hline Logistic EuroSCORE (\%) & $17.3 \pm 10.2$ & $20.2 \pm 10.2$ & $26.9 \pm 12.6$ & $<0.01$ \\
\hline EuroSCORE II (\%) & $5.3 \pm 4.2$ & $7.3 \pm 4.1$ & $10.3 \pm 5.7$ & $<0.01$ \\
\hline STS score (\%) & $5.2 \pm 4.3$ & $7.7 \pm 5.2$ & $9.9 \pm 6.3$ & $<0.01$ \\
\hline Creatinine clearance $(\mathrm{ml} / \mathrm{min})$ & $76.7 \pm 26.9$ & $52.0 \pm 13.2$ & $34.8 \pm 11.2$ & $<0.01$ \\
\hline Creatinine $(\mu \mathrm{mol} / \mathrm{l})$ & $78.2 \pm 19.1$ & $100.7 \pm 24.1$ & $168.3 \pm 124.5$ & $<0.01$ \\
\hline CKD 4 & $0(0)$ & $0(0)$ & $64(28)$ & - \\
\hline \multicolumn{5}{|l|}{ Echocardiographic data } \\
\hline LVEF (\%) & $57.6 \pm 10.5$ & $53.1 \pm 14.5$ & $43.7 \pm 14.7$ & $<0.01$ \\
\hline Low EF & $21(9)$ & $62(26)$ & $110(48)$ & $<0.01$ \\
\hline $\operatorname{AVA}\left(\mathrm{cm}^{2}\right)$ & $0.66 \pm 0.14$ & $0.62 \pm 0.14$ & $0.62 \pm 0.15$ & $<0.01$ \\
\hline Mean gradient $(\mathrm{mmHg})$ & $48.1 \pm 16.4$ & $47.7 \pm 16.6$ & $45.2 \pm 17.5$ & 0.13 \\
\hline AR grade $(0-4)$ & $0.86 \pm 0.78$ & $0.88 \pm 0.74$ & $0.91 \pm 0.73$ & 0.77 \\
\hline $\mathrm{PAP}(\mathrm{mmHg})$ & $45.7 \pm 12.2$ & $47.2 \pm 14.9$ & $51.0 \pm 13.7$ & $<0.01$ \\
\hline
\end{tabular}

Data given as $\mathrm{n}(\%)$ or mean $\pm \mathrm{SD}$. ACEF, age, creatinine clearance, ejection fraction; $\mathrm{AR}$, aortic regurgitation; $\mathrm{AVA}$, aortic valve area; $\mathrm{BMI}$, body mass index; BSA, body surface area; CABG, coronary artery bypass grafting; CKD, chronic kidney disease; COPD, chronic obstructive pulmonary disease; EF, ejection fraction; LVEF, left ventricle EF; MI, myocardial infarction; MR, mitral regurgitation; NYHA, New York Heart Association; PAP, pulmonary artery pressure; $\mathrm{PCl}$, percutaneous coronary intervention.

included in the dedicated TAVI database. Patients with severe AS were considered candidates for TAVI if they were deemed ineligible or high risk for SAVR. The decision to proceed with TAVI was made by a dedicated heart team composed of experienced clinical and interventional cardiologists, cardiovascular surgeons and anesthesiologists. Between October 2006 and May 2013, a total of 703 patients were included. Written informed consent was obtained from all patients.

The STS score, LES, ES II and ACEFmodif score were assessed. The ACEFmodif score was calculated using the following formula: age $/ \mathrm{EF}+1$ point for every $10-\mathrm{ml} / \mathrm{min}$ reduction in creatinine clearance $<60 \mathrm{ml} / \mathrm{min} / 1.73 \mathrm{~m}^{2}$ (up to a maximum of 6 points), as described previously. ${ }^{10}$

\section{Vascular Access and Valve Selection}

Patients were selected to undergo TAVI via the transfemoral (TF) approach or alternative approaches depending on the size, calcification and tortuosity of the ilio-femoral arterial access. In most instances, the type of valve prosthesis was selected according to the team's and the main operator's preference. The trans-subclavian (SC) or transaortic approach (TAo) was used as an alternative in cases of unsuitable femoral arterial access in recipients of the CoreValve, and the transapical (TA), trans-subclavian, or transaortic route as the alternative to suboptimal femoral access with the Edwards valve.

\section{Procedures}

Aspirin (75-160 mg) and clopidogrel (75 mg) daily were recommended prior to TAVI. A loading dose of clopidogrel (300$600 \mathrm{mg}$ ) was administered to patients who were not already on clopidogrel before or immediately after the procedure. Only 1 antiplatelet therapy, usually aspirin, was associated with warfarin in the majority of patients on long-term anticoagulant therapy. A bolus of heparin $(70 \mathrm{IU} / \mathrm{kg})$ was administered at the start of the procedure to achieve an activated clotting time of $250-300 \mathrm{~s}$, and the activated clotting time was measured every $30 \mathrm{~min}$ thereafter. All procedures were performed by an experienced team according to the standard operating procedures, as previously described. ${ }^{13}$

\section{Post-Procedural Care}

All patients were observed in the intensive care unit for at least 


\begin{tabular}{|c|c|c|c|c|}
\hline & $\begin{array}{l}\text { Low ACEF modif } \\
(0.6-2.1)(n=236)\end{array}$ & $\begin{array}{l}\text { Middle ACEF modif } \\
(2.2-3.8)(n=237)\end{array}$ & $\begin{array}{l}\text { High ACEF modif } \\
(3.9-9.6)(n=230)\end{array}$ & P-value \\
\hline Type of valve & & & & 0.20 \\
\hline Edwards valve & $194(82)$ & $185(78)$ & $175(76)$ & \\
\hline CoreValve or another valve & $42(18)$ & $52(22)$ & $55(24)$ & \\
\hline \multicolumn{5}{|l|}{ Approach route } \\
\hline TF approach & $118(50)$ & $123(52)$ & $142(62)$ & 0.01 \\
\hline TA approach & $37(16)$ & $45(19)$ & $34(15)$ & 0.46 \\
\hline TAo approach & $77(33)$ & $61(26)$ & $48(21)$ & 0.01 \\
\hline Valve size $(\mathrm{mm})$ & $26.1 \pm 2.2$ & $26.1 \pm 2.3$ & $26.2 \pm 2.4$ & 0.81 \\
\hline Early experience & $15(6)$ & $19(8)$ & $16(7)$ & 0.77 \\
\hline \multicolumn{5}{|l|}{ Post-procedural variables } \\
\hline Length of hospital stay (days) & $11.6 \pm 7.7$ & $11.8 \pm 8.8$ & $11.7 \pm 10.2$ & 0.98 \\
\hline Procedural success & $221(94)$ & $210(89)$ & $213(93)$ & 0.13 \\
\hline 30-day mortality & $21(8)$ & $33(13)$ & $42(18)$ & $<0.01$ \\
\hline 30-day combined safety endpoint & $35(15)$ & $53(23)$ & $58(26)$ & $<0.01$ \\
\hline Need for $\mathrm{PCl}$ & $4(2)$ & $2(1)$ & $5(2)$ & 0.51 \\
\hline Cerebrovascular accidents & $2(1)$ & $7(3)$ & $9(4)$ & 0.18 \\
\hline AKI & $25(10)$ & $25(10)$ & $49(22)$ & $<0.01$ \\
\hline Major vascular complication & $14(6)$ & $28(12)$ & $23(10)$ & 0.08 \\
\hline Major bleeding & 7 (3) & $7(3)$ & $4(2)$ & 0.87 \\
\hline $\mathrm{RBC}$ transfusion & $61(28)$ & $64(29)$ & $67(31)$ & 0.85 \\
\hline Pacemaker implantation & $20(9)$ & $23(10)$ & $20(9)$ & 0.85 \\
\hline 2 valve implantation & $7(3)$ & $12(5)$ & $5(2)$ & 0.20 \\
\hline Post-procedural AR $\geq$ grade 2 & $30(13)$ & $47(20)$ & $56(25)$ & $<0.01$ \\
\hline Need for any cardiac surgery & $5(2)$ & $7(3)$ & $5(2)$ & 0.63 \\
\hline
\end{tabular}

Data given as $\mathrm{n}(\%)$ or mean $\pm \mathrm{SD}$. AKI, acute kidney injury; RBC, red blood cell; TA, transapical; TAo, transaortic; TF, transfemoral. Other abbreviations as in Table 1.

$24 \mathrm{~h}$ after Edwards valve implantation or $72 \mathrm{~h}$ after CoreValve implantation (patients without previous pacemaker). Dual antiplatelet therapy was continued for 1-6 months and, thereafter, aspirin was continued indefinitely.

\section{Follow-up}

After the procedure, all patients were assessed by a physician at 1, 3, 6 and 12 months postoperatively and annually thereafter. Additional follow-up data were collected via telephone interviews and contact with the patients' family physicians.

\section{Endpoints}

The main endpoints of this study were the incidence of acute kidney injury (AKI) and all-cause mortality at 30-day and 1-year follow-up. All adverse events were assessed according to the Valve Academic Research Consortium (VARC) criteria. Adverse events were prospectively collected by the research technicians and validated by the cardiologists.

\section{Statistical Analysis}

All statistical analysis was performed using SPSS version 21.0 (Chicago, IL, USA). Continuous variables are expressed as mean $\pm \mathrm{SD}$ or with the corresponding interquartile range (IQR). Dichotomous variables are expressed as counts and percentages. Comparison between 3 groups was performed using Pearson's bivariate test and the chi-squared test for categorical covariates, and 1-way ANOVA for continuous covariates. Univariate logistic regression analysis was performed to investigate the factors independently associated with AKI. Subsequently, multivariate analysis was performed using variables with
$\mathrm{P}<0.10$ on univariate analysis in order to examine their independent association with AKI. Survival analysis was performed using the Kaplan-Meier method. Univariate Cox regression analysis was performed to examine the variables related to 1 -year cumulative mortality. Subsequently, multivariate analysis was performed using variables with $\mathrm{P}<0.10$ on univariate analysis in order to examine their independent association with 1-year cumulative mortality. Receiver operating characteristic (ROC) analysis with the area under curve (AUC) as a measurement of accuracy was used to compare the accuracy between different risk scores. $\mathrm{P}<0.05$ was considered significant.

\section{Results}

\section{Baseline Characteristics and Post-Procedural Outcome}

Of the 703 study patients, 236 were in the lowest tertile (ACEFmodif score 0.6-2.1), 237 in the middle tertile (ACEFmodif score 2.2-3.8), and 230 in the highest tertile (ACEFmodif score 3.9-9.6). Baseline characteristics are given in Table 1. Variations in baseline characteristics between the 3 study groups resulted in significant differences in the mean logistic EuroSCORE (17.3 vs. 20.2 vs. 26.9, $\mathrm{P}<0.001)$, EuroSCORE II (5.3 vs. 7.3 vs. 10.3, $\mathrm{P}<0.001)$ and STS score (5.2 vs. 7.7 vs. $9.9, \mathrm{P}<0.001$ ). Procedural characteristics are listed in Table 2. The Edwards valve was used in 554 patients (78\%) and other valves, mainly the CoreValve, in 149 (22\%). Although there were no significant differences between the 3 groups regarding the use of the TA approach (16\% vs. $19 \%$ vs. $15 \%, \mathrm{P}=0.46)$, there was a significant difference between the groups with respect to the use of the TAo approach (33\% vs. 
Table 3. Predictors of AKI

\begin{tabular}{|c|c|c|c|c|c|c|}
\hline & \multicolumn{3}{|c|}{ Univariate analysis } & \multicolumn{3}{|c|}{ Multivariate analysis } \\
\hline & OR & $95 \% \mathrm{Cl}$ & P-value & OR & $95 \% \mathrm{Cl}$ & P-value \\
\hline Diabetes & 1.18 & $0.72-1.93$ & 0.49 & & & \\
\hline End-stage renal disease & 3.41 & $1.11-10.03$ & 0.03 & 1.72 & $0.48-6.43$ & 0.38 \\
\hline STS score (per 1-point increase) & 1.00 & $0.96-1.03$ & 0.97 & & & \\
\hline EuroSCORE (per 1-point increase) & 1.01 & $0.99-1.03$ & 0.10 & & & \\
\hline EuroSCORE II (per 1-point increase) & 1.04 & $1.00-1.08$ & 0.04 & 1.01 & $0.96-1.06$ & 0.61 \\
\hline ACEFmodif (per 1-point increase) & 1.25 & $1.11-1.41$ & $<0.01$ & 1.21 & $1.04-1.41$ & 0.01 \\
\hline
\end{tabular}

End-stage renal disease, creatinine clearance $<15 \mathrm{ml} / \mathrm{min} / 1.73 \mathrm{~m}^{2}$. ACEFmodif, modified ACEF score; EuroSCORE, European System for Cardiac Operative Risk Evaluation; STS, Society of Thoracic Surgeons. Other abbreviations as in Tables 1,2.

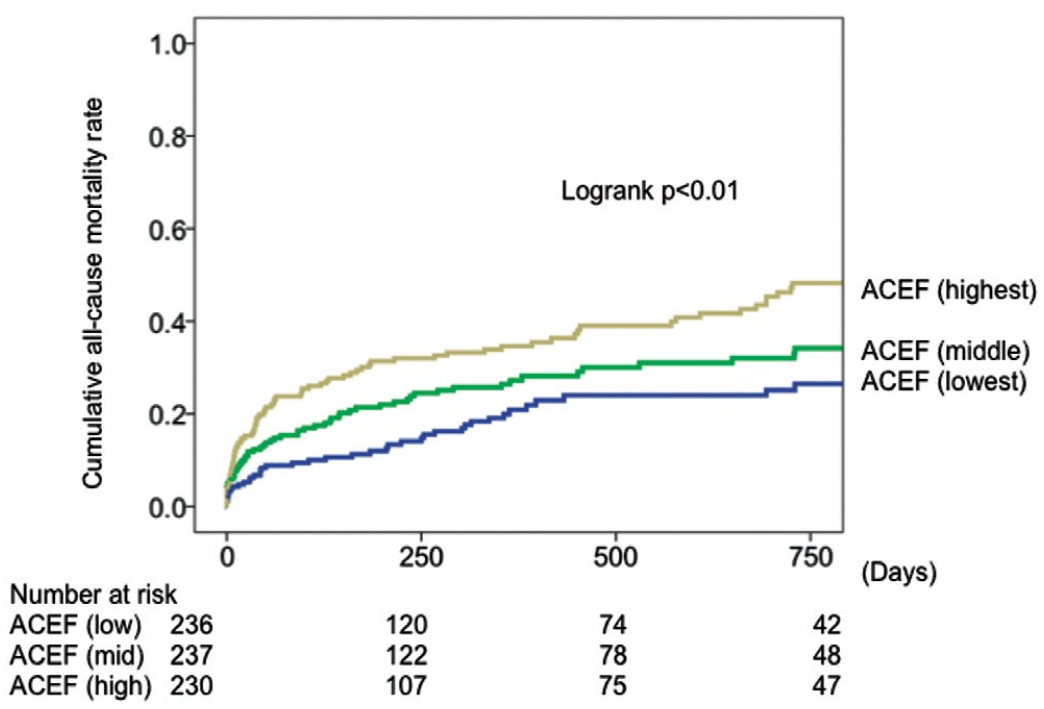

Figure 1. Cumulative all-cause mortality vs. modified age, creatinine clearance, ejection fraction (ACEF) tertile.

$26 \%$ vs. $21 \%, \mathrm{P}=0.01)$ and TF approach ( $50 \%$ vs. $52 \%$ vs. $62 \%$, $\mathrm{P}=0.01)$. There was no significant difference between the 3 groups in the number of early-experience cases, which was defined as the first 50 cases. While no significant differences were observed in the procedural success rates between the 3 groups, the 30 -day mortality rate ( $8 \%$ vs. $13 \%$ vs. $18 \%, \mathrm{P}<0.01)$, 30 -day combined safety endpoints $(15 \%$ vs. $23 \%$ vs. $26 \%$, $\mathrm{P}<0.01)$, and the incidence of AKI $(10 \%$ vs. $10 \%$ vs. $22 \%$, $\mathrm{P}<0.01)$, significantly increased with ACEFmodif tertile.

\section{Predictors of AKI}

Predictors of AKI were evaluated on logistic regression analysis (Table 3). On univariate analysis, diabetes, creatinine clearance $\left(<15 \mathrm{ml} / \mathrm{min} / 1.73 \mathrm{~m}^{2}\right)$ and ACEFmodif were significant predictors of AKI. On multivariate logistic regression, ACEFmodif score was the only independent predictor of AKI.

\section{Impact of ACEFmodif Score on Mid-Term Mortality After TAVI} The median follow-up period of this cohort was 406 days (IQR, 35-730). Follow-up at 1 year was complete in 422 (60\%) of the 703 study patients. Cumulative all-cause mortality rates at 1 year according to ACEFmodif tertile were $22 \%, 28 \%$, and $36 \%$, respectively. Cumulative all-cause mortality rates were calculated using the Kaplan-Meier method and compared using log-rank test (Figure 1). The mortality rate in the low ACEFmodif tertile was significantly lower in comparison with the middle ACEFmodif tertile and high ACEFmodif tertile. Similarly, cumulative cardiovascular mortality rates at 1 year according to ACEFmodif tertile were $14 \%, 17 \%$, and $25 \%$, respectively. Cumulative cardiovascular death rate in the low ACEFmodif tertile was significantly lower in comparison with the middle ACEFmodif tertile and high ACEFmodif tertile (Figure 2).

\section{Predictors of 1-Year Cumulative Mortality}

Predictors of 1-year cumulative mortality were evaluated on Cox regression analysis (Table 4). On univariate analysis, TA approach, post-procedural aortic regurgitation (AR; $\geq$ grade 2), STS score, EuroSCORE, EuroSCORE II and ACEFmodif score were significant predictors of 1-year mortality. On multivariate Cox regression, TA approach, post-procedural AR ( $\geq$ grade 2) and ACEFmodif score were independent predictors of 1-year cumulative mortality (Figure 3 ).

\section{Risk Score Accuracy}

According to AUC, all risk scores poorly predicted AKI, 30-day and 1-year cumulative mortality (Figure 4), but ACEFmodif had better predictive value for AKI compared with other risks and the same predictive value for 30-day and 1-year mortality as other risk models. 


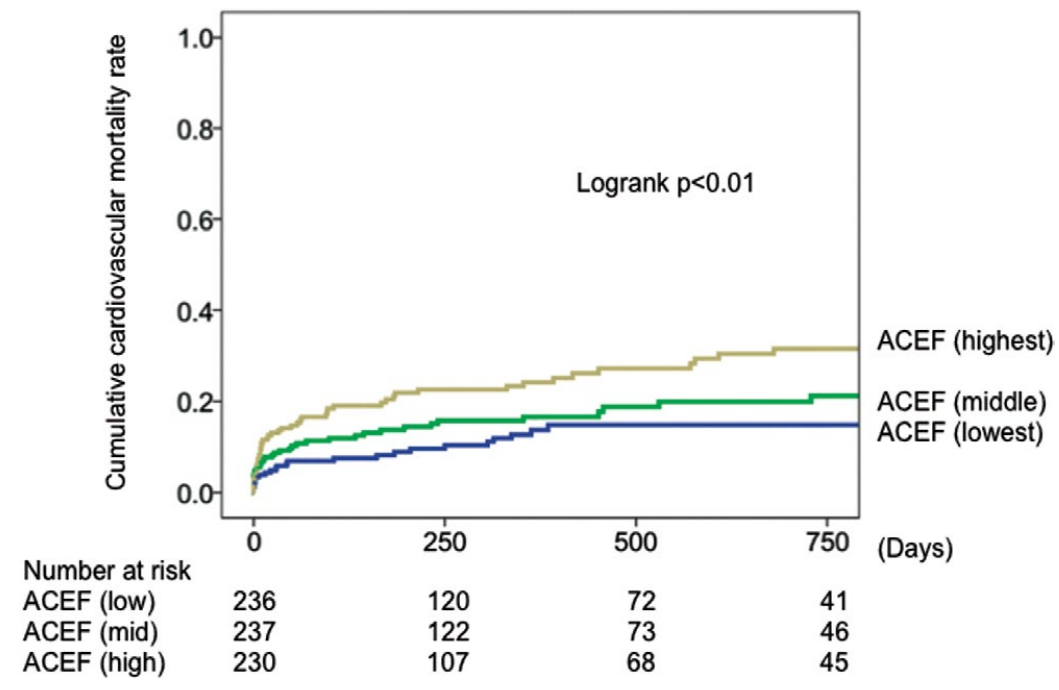

Figure 2. Cumulative cardiovascular mortality vs. modified age, creatinine clearance, ejection fraction (ACEF) tertile.

\begin{tabular}{|lrcc|}
\hline \multicolumn{2}{|l|}{ Table 4. Univariate Indicators of 1-Year Cumulative Mortality } & & P-value \\
Low EF & HR & $95 \%$ Cl & 0.19 \\
COPD & 1.25 & $0.89-1.75$ & 0.11 \\
Male & 1.31 & $0.94-1.82$ & 0.40 \\
End-stage renal disease & 1.14 & $0.83-1.56$ & 0.54 \\
TA approach & 1.36 & $0.50-3.68$ & $<0.01$ \\
TF approach & 1.71 & $1.21-2.42$ & 0.30 \\
Post-procedural AR $\geq$ grade 2 & 0.84 & $0.62-1.15$ & $<0.01$ \\
STS score (per 1-point increase) & 1.68 & $1.18-2.39$ & $<0.01$ \\
EuroSCORE (per 1-point increase) & 1.03 & $1.01-1.05$ & $<0.01$ \\
EuroSCORE II (per 1-point increase) & 1.02 & $1.00-1.03$ & $<0.01$ \\
ACEFmodif (per 1-point increase) & 1.05 & $1.03-1.08$ & $<0.01$ \\
\hline
\end{tabular}

End-stage renal disease, creatinine clearance $<15 \mathrm{ml} / \mathrm{min} / 1.73 \mathrm{~m}^{2}$. Abbreviations as in Tables $1-3$.

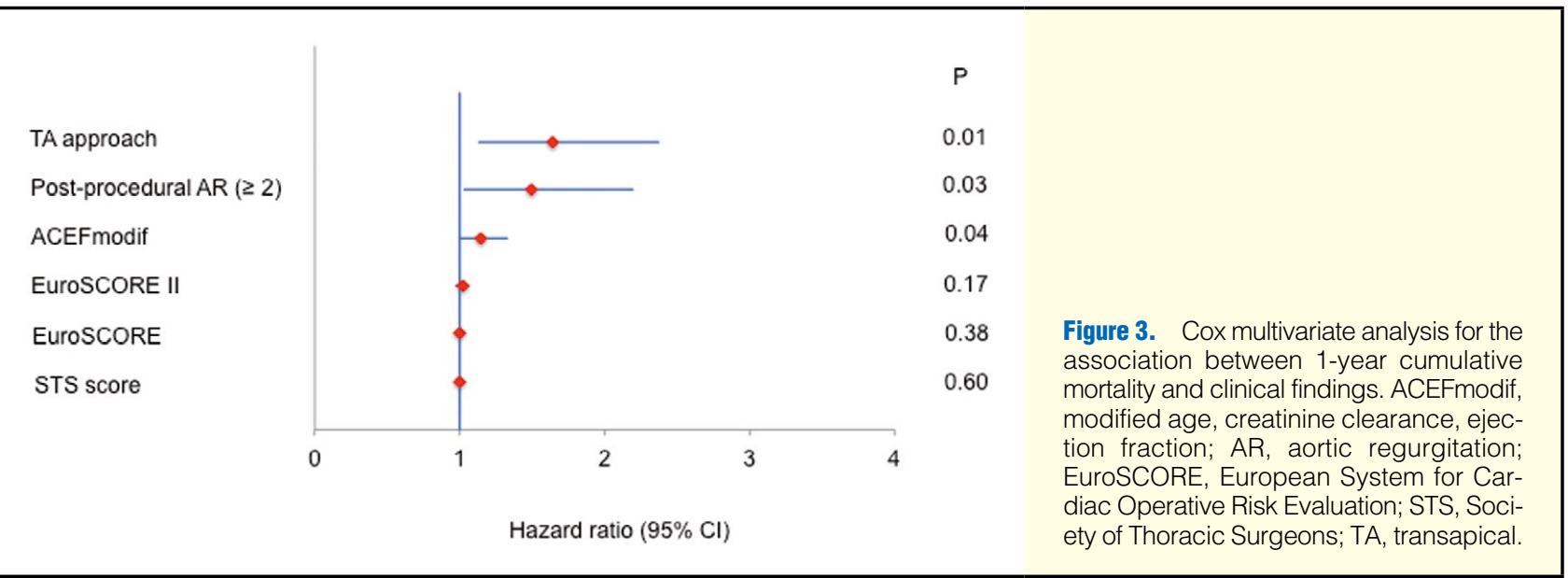

\section{Prediction of AKI and Mortality According to Access}

According to AUC, ACEFmodif had a better predictive value for 30-day mortality compared with other risk scores in the non-TF cohort, although ES II had a better predictive value for 30-day mortality compared with other risk scores in the TF cohort. Furthermore, ACEFmodif also had the same predictive value (albeit poor), for AKI and 1-year mortality as other risk models both in the TF cohort and non-TF cohort (TableS1). 
A

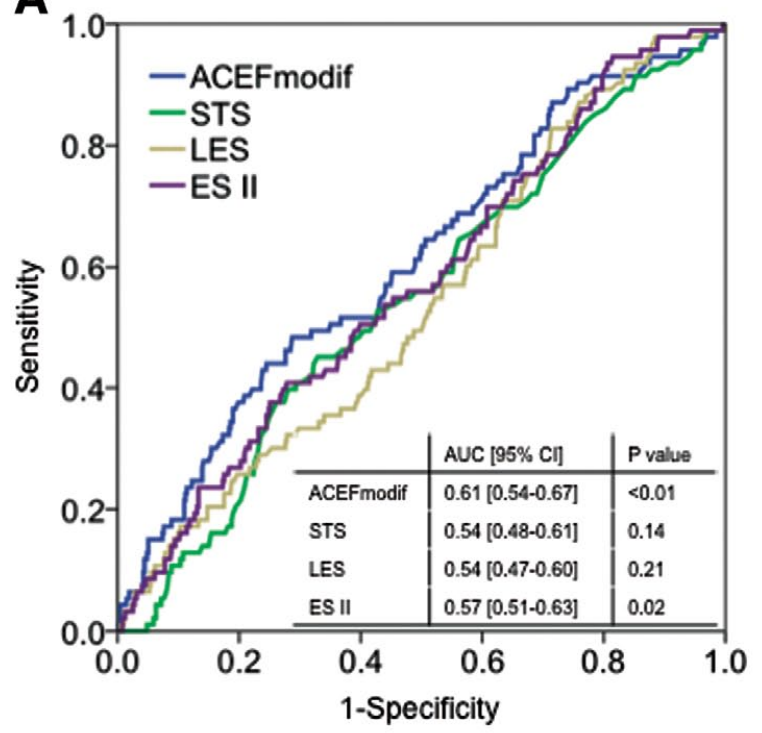

B

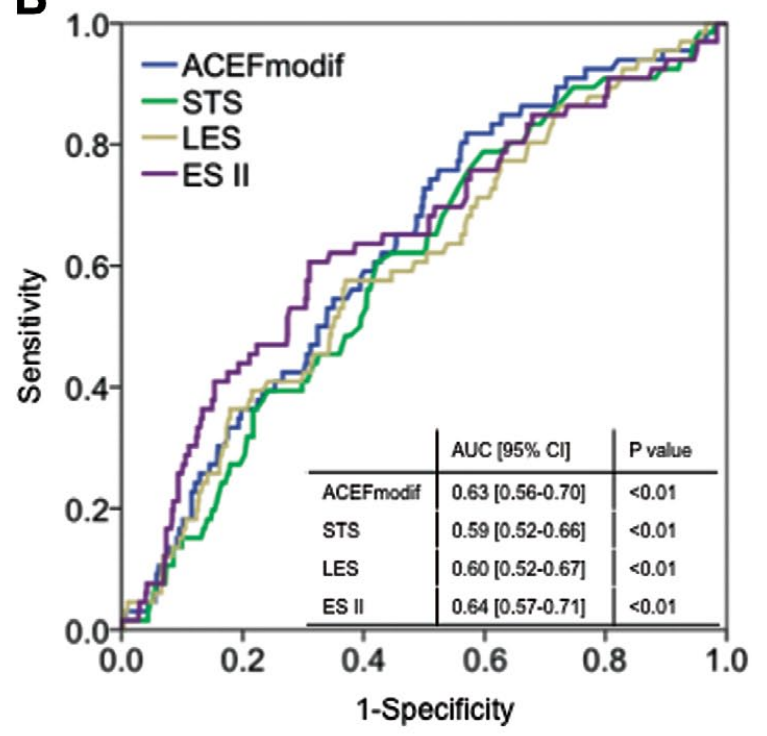

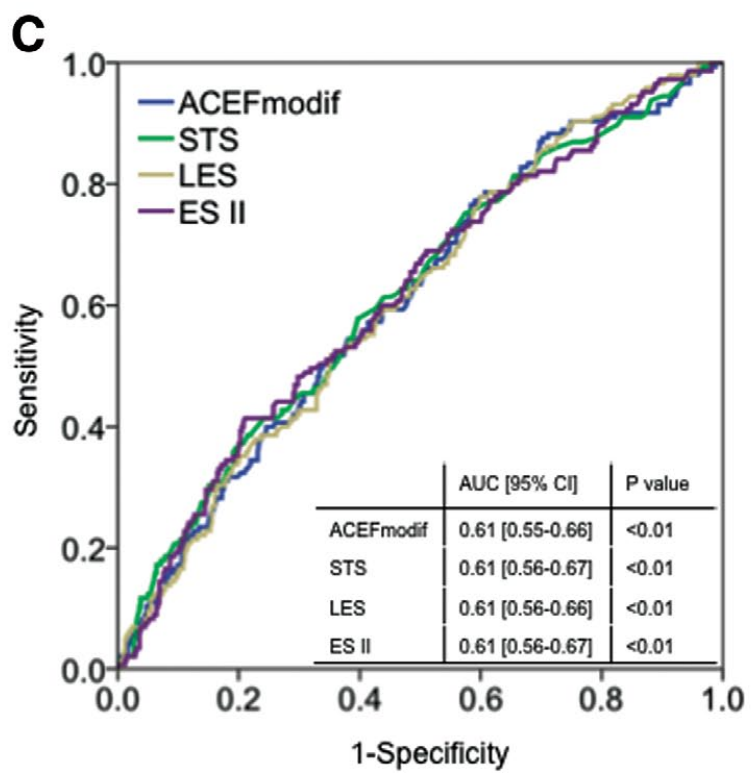

Figure 4. (A) Receiver operating characteristic curves for modified age, creatinine clearance, ejection fraction (ACEFmodif) score, Society of Thoracic Surgeons (STS) score, Logistic European System for Cardiac Operative Risk Evaluation (LES) and European System for Cardiac Operative Risk Evaluation Score (ES) II for (A) AKI, (B) 30-day mortality and (C) 1-year mortality.

\section{Discussion}

The present study indicates that ACEFmodif score might provide useful information for predicting AKI, 30-day and 1-year mortality in patients undergoing TAVI. This simple scoring method was tested in patients undergoing $\mathrm{CABG}$ or PCI and proved valuable in predicting outcome. So far, only 1 study has investigated the usefulness of ACEF score in predicting outcome in TAVI patients. ${ }^{14}$ To the best of our knowledge, the present study is the first to show that ACEFmodif score has the potential to predict outcome in TAVI patients. In addition, ACEF score has been reported to be a good predictor of AKI after PCI. ${ }^{15}$ The present study is also the first to suggest that ACEFmodif score is a good predictor of AKI after TAVI.

ACEF score is a very simple scoring tool that includes only 3 variables. These 3 variables have been shown to be associated with the prognosis of CABG and PCI, ${ }^{16-18}$ and have also been investigated in TAVI patients. With respect to age, a number of studies have shown that TAVI can be performed in elderly patients, but the relationship between age and prognosis is still under debate. ${ }^{19,20}$ With regard to EF, the majority of studies have shown that low EF is a predictor of poor outcome..$^{21,22}$ Nevertheless, 1 study has shown that TAVI is associated with earlier recovery of EF and good outcome. ${ }^{23}$ These contrasting findings account for the fact that the relationship between low EF and the TAVI outcome has not yet been elu- 
cidated. With regard to renal insufficiency, many studies have shown that pre-procedural renal failure is a predictor of 30-day and 1-year mortality. ${ }^{24}$

The use of multiple clinical variables should improve the accuracy of risk models, although this accuracy may ultimately be negatively affected by the desire to create the perfect model. ${ }^{25}$ The LES, ES II and STS scores have often been used for risk assessment before TAVI, ${ }^{6,26,27}$ but even though they include many variables, these scores are not very reliable (AUC<0.7). ${ }^{26}$ The ACEFmodif score is a very simple scoring tool that includes only 3 variables and seems to be quite easy to use. This score has been shown to be as accurate as the LES score, which includes 17 clinical variables, in patients undergoing elective CABG. ${ }^{7}$ The present study also showed that ACEFmodif score has a superior predictive value for AKI compared with other risk models.

In the present study, ACEFmodif score had better predictive value for AKI compared with other risk models both in the TF and non-TF cohort, as well as better predictive value for 30-day mortality compared with other risk scores in the non-TF cohort. In addition, ACEFmodif had the same predictive value for midterm mortality as other risk models both in the TF and non-TF cohorts.

This study showed that not only ACEFmodif score, but also the TA approach was a predictor of 1-year cumulative mortality. Gilard et al. also reported in the FRANCE 2 study that the TA approach was significantly associated with increased 1-year mortality. ${ }^{28}$ The TAo approach is currently proposed as an alternative to the TA approach for patients in whom the TF approach is not feasible. ${ }^{29}$ Furthermore, this study showed that postprocedural AR ( $\geq$ grade 2 ) was a predictor of 1-year mortality. Some reports have shown that even mild AR is associated with poorer outcome. ${ }^{6,30,31}$ Next-generation devices designed to reduce post-procedural AR are now being used.

ACEFmodif score uses creatinine clearance instead of serum creatinine level. Among patients undergoing $\mathrm{CABG}$, creatinine clearance has been shown to be a better predictor when compared with serum creatinine. ${ }^{32,33}$ In addition, ACEFmodif score has been reported to increase the accuracy of the original ACEF score among PCI patients. Furthermore, the stage of chronic kidney disease on the basis of the estimated glomerular filtration rate has been shown to be strongly related to TAVI outcome. ${ }^{24}$ Thus, ACEFmodif is potentially a better predictor than the original ACEF score in TAVI patients.

ACEF score has been reported to be a good predictor of AKI and mortality after PCI. ${ }^{10,15}$ Consequently, this study focused on the predictive value of ACEFmodif for AKI and mortality. The predictive value of ACEFmodif on cerebrovascular events, myocardial infarction and life-threatening bleeding after TAVI were also evaluated on logistic regression analysis. ACEFmodif, however, was not a good predictor of cerebrovascular events (OR, 1.22; 95\% CI: 0.95-1.57, $\mathrm{P}=0.11)$, myocardial infarction (OR, 0.80; 95\% CI: 0.37-1.75, P=0.59) and life-threatening bleeding (OR, 0.99; 95\% CI: 0.83-1.17, $\mathrm{P}=0.89$ ).

This study shows that a simple and objective score such as ACEFmodif is very useful for predicting outcome in patients undergoing TAVI. It is important to emphasize, however, that currently available risk scores including ACEF score, LES, ES II and STS score cannot provide a thorough assessment of the risks before TAVI. Risk evaluation should be carried out for each individual case by the heart team, which consists of experienced interventional cardiologists, cardiac surgeons, anesthetists and imaging specialists as well as geriatricians in difficult cases.

\section{Study Limitations}

The present study has several limitations that should be addressed. First, this was a retrospective observational singlecenter study carried out in a limited number of patients. Further studies with larger cohorts and multiple-center analysis are required to confirm our result. Second, the risk-adjusted multivariate model in this study did not include all possible residual variables because of the limited size of the study. Third, the rate of 1-year follow-up was $60 \%$ in all patients. This impaired the validity of the identification of predictors of 1-year mortality. The results need further confirmation. Finally, the mean follow-up period was 406 days, and longterm follow-up is needed to investigate the impact of ACEF score on long-term outcome.

\section{Conclusions}

ACEFmodif score may provide useful information for predicting AKI, 30-day and 1-year mortality in patients undergoing TAVI, but the present results need further confirmation.

\section{Acknowledgments}

The authors thank Mrs Catherine Dupic for her assistance with the manuscript. This study was supported, in part, by research grants from the Japanese Circulation Society (to T.A.).

\section{Conflicts of Interest}

T.L. is a proctor for transfemoral-TAVI for Edwards Lifesciences, and a consultant for Symetis and Direct Flow Medical. M.R. is a proctor for transapical-TAVI for Edwards Lifesciences. K.H. is a proctor for transfemoral-TAVI for Edwards Lifesciences. B.C. is a consultant for Medtronic.

\section{References}

1. Zahn R, Gerckens U, Grube E, Linke A, Sievert H, Eggebrecht H, et al. Transcatheter aortic valve implantation: First results from a multicentre real-world registry. Eur Heart J 2011; 32: 198-204.

2. Moat NE, Ludman P, de Belder MA, Bridgewater B, Cunningham $\mathrm{AD}$, Young CP, et al. Long-term outcomes after transcatheter aortic valve implantation in high-risk patients with severe aortic stenosis: The U.K. TAVI (United Kingdom Transcatheter Aortic Valve Implantation) registry. J Am Coll Cardiol 2011; 58: 2130-2138.

3. Webb J, Cribier A. Percutaneous transarterial aortic valve implantation: What do we know? Eur Heart J 2011; 32: 140-147.

4. Kobayashi J. Changing strategy for aortic stenosis by transcatheter valve treatment in Japan. Circ J 2013; 77: 309-310.

5. Lefevre T, Kappetein AP, Wolner E, Nataf P, Thomas M, Schachinger $\mathrm{V}$, et al. One year follow-up of the multi-centre European PARTNER transcatheter heart valve study. Eur Heart J 2011; 32: 148-157.

6. Kodali SK, Williams MR, Smith CR, Svensson LG, Webb JG, Makkar RR, et al. Two-year outcomes after transcatheter or surgical aortic-valve replacement. $N$ Engl J Med 2012; 366: 1686-1695.

7. Ranucci M, Castelvecchio S, Menicanti L, Frigiola A, Pelissero G. Risk of assessing mortality risk in elective cardiac operations: Age, creatinine, ejection fraction, and the law of parsimony. Circulation 2009; 119: 3053-3061.

8. Wykrzykowska JJ, Garg S, Onuma Y, de Vries T, Goedhart D, Morel MA, et al. Value of age, creatinine, and ejection fraction (ACEF score) in assessing risk in patients undergoing percutaneous coronary interventions in the 'All-Comers' LEADERS trial. Circ Cardiovasc Interv 2011; 4: 47-56.

9. Biondi-Zoccai G, Romagnoli E, Castagno D, Sheiban I, De Servi S, Tamburino C, et al. Simplifying clinical risk prediction for percutaneous coronary intervention of bifurcation lesions: The case for the ACEF (age, creatinine, ejection fraction) score. EuroIntervention 2012; 8: $359-367$.

10. Garg S, Sarno G, Garcia-Garcia HM, Girasis C, Wykrzykowska J, Dawkins KD, et al. A new tool for the risk stratification of patients with complex coronary artery disease: The clinical SYNTAX score. Circ Cardiovasc Interv 2010; 3: 317-326.

11. Capodanno D, Marcantoni C, Ministeri M, Dipasqua F, Zanoli L, Rastelli S, et al. Incorporating glomerular filtration rate or creatinine 
clearance by the modification of diet in renal disease equation or the Cockcroft-Gault equations to improve the global accuracy of the age, creatinine, ejection fraction [ACEF] score in patients undergoing percutaneous coronary intervention. Int J Cardiol 2013; 168: $396-$ 402.

12. Capodanno D. Beyond the SYNTAX score: Advantages and limitations of other risk assessment systems in left main percutaneous coronary intervention. Circ J 2013; 77: 1131-1138.

13. Hayashida K, Lefevre T, Chevalier B, Hovasse T, Romano M, Garot $\mathrm{P}$, et al. Transfemoral aortic valve implantation new criteria to predict vascular complications. JACC Cardiovasc Interv 2011; 4: 851-858.

14. D'Ascenzo F, Ballocca F, Moretti C, Barbanti M, Gasparetto V, Mennuni $\mathrm{M}$, et al. Inaccuracy of available surgical risk scores to predict outcomes after transcatheter aortic valve replacement. J Cardiovasc Med (Hagerstown) 2013; 14: 894-898.

15. Ando G, Morabito G, de Gregorio C, Trio O, Saporito F, Oreto G. The ACEF score as predictor of acute kidney injury in patients undergoing primary percutaneous coronary intervention. Int J Cardiol 2013; 168: $4386-4387$.

16. Feldman DN, Gade CL, Slotwiner AJ, Parikh M, Bergman G, Wong SC, et al. Comparison of outcomes of percutaneous coronary interventions in patients of three age groups $(<60,60$ to 80 , and $>80$ years $)$ (from the New York State Angioplasty Registry). Am J Cardiol 2006; 98: $1334-1339$.

17. Shaw JA, Andrianopoulos N, Duffy S, Walton AS, Clark D, Lew R, et al. Renal impairment is an independent predictor of adverse events post coronary intervention in patients with and without drug-eluting stents. Cardiovasc Revasc Med 2008; 9: 218-223.

18. Keelan PC, Johnston JM, Koru-Sengul T, Detre KM, Williams DO, Slater J, et al. Comparison of in-hospital and one-year outcomes in patients with left ventricular ejection fractions $<$ or $=40 \%, 41 \%$ to $49 \%$, and $>$ or $=50 \%$ having percutaneous coronary revascularization. Am J Cardiol 2003; 91: $1168-1172$.

19. Yamamoto M, Meguro K, Mouillet G, Bergoend E, Monin JL, Lim $\mathrm{P}$, et al. Comparison of effectiveness and safety of transcatheter aortic valve implantation in patients aged $\geq 90$ years versus $<90$ years. $A m$ J Cardiol 2012; 110: 1156-1163.

20. Yamamoto M, Mouillet G, Meguro K, Gilard M, Laskar M, Eltchaninoff $\mathrm{H}$, et al. Clinical results of transcatheter aortic valve implantation in octogenarians and nonagenarians: Insights from the FRANCE-2 registry. Ann Thorac Surg 2014; 97: 29-36.

21. van der Boon RM, Nuis RJ, Van Mieghem NM, Benitez LM, van Geuns RJ, Galema TW, et al. Clinical outcome following transcatheter aortic valve implantation in patients with impaired left ventricular systolic function. Catheter Cardiovasc Interv 2012; 79: 702-710.

22. O'Sullivan CJ, Stortecky S, Heg D, Pilgrim T, Hosek N, Buellesfeld $\mathrm{L}$, et al. Clinical outcomes of patients with low-flow, low-gradient, severe aortic stenosis and either preserved or reduced ejection fraction undergoing transcatheter aortic valve implantation. Eur Heart $J$ 2013; 34: 3437-3450.
23. Bauer F, Coutant V, Bernard M, Stepowski D, Tron C, Cribier A, et al. Patients with severe aortic stenosis and reduced ejection fraction: Earlier recovery of left ventricular systolic function after transcatheter aortic valve implantation compared with surgical valve replacement. Echocardiography 2013; 30: 865-870.

24. Yamamoto M, Hayashida K, Mouillet G, Hovasse T, Chevalier B, Oguri A, et al. Prognostic value of chronic kidney disease after transcatheter aortic valve implantation. J Am Coll Cardiol 2013; 62: $869-877$.

25. Concato J, Feinstein AR, Holford TR. The risk of determining risk with multivariable models. Ann Intern Med 1993; 118: 201-210.

26. Watanabe Y, Hayashida K, Lefevre T, Chevalier B, Hovasse T, Romano M, et al. Is EuroSCORE II better than EuroSCORE in predicting mortality after transcatheter aortic valve implantation? Catheter Cardiovasc Interv 2013; 81: 1053-1060.

27. Durand E, Borz B, Godin M, Tron C, Litzler PY, Bessou JP, et al. Performance analysis of EuroSCORE II compared to the original logistic EuroSCORE and STS scores for predicting 30-day mortality after transcatheter aortic valve replacement. Am J Cardiol 2013; 111: 891-897.

28. Gilard M, Eltchaninoff H, Iung B, Donzeau-Gouge P, Chevreul K, Fajadet J, et al. Registry of transcatheter aortic-valve implantation in high-risk patients. $N$ Engl J Med 2012; 366: 1705-1715.

29. Hayashida K, Romano M, Lefevre T, Chevalier B, Farge A, Hovasse $\mathrm{T}$, et al. The transaortic approach for transcatheter aortic valve implantation: A valid alternative to the transapical access in patients with no peripheral vascular option: A single center experience. Eur J Cardiothorac Surg 2013; 44: 692-700.

30. Hayashida K, Lefevre T, Chevalier B, Hovasse T, Romano M, Garot $\mathrm{P}$, et al. Impact of post-procedural aortic regurgitation on mortality after transcatheter aortic valve implantation. JACC Cardiovasc Interv 2012; 5: $1247-1256$.

31. Werner N, Sinning JM. Aortic regurgitation after transcatheter aortic valve replacement. Circ J 2014; 78: 811-818.

32. Walter J, Mortasawi A, Arnrich B, Albert A, Frerichs I, Rosendahl $\mathrm{U}$, et al. Creatinine clearance versus serum creatinine as a risk factor in cardiac surgery. BMC Surg 2003; 3: 4.

33. Noyez L, Plesiewicz I, Verheugt FW. Estimated creatinine clearance instead of plasma creatinine level as prognostic test for postoperative renal function in patients undergoing coronary artery bypass surgery. Eur J Cardiothorac Surg 2006; 29: 461-465.

\section{Supplementary Files}

Supplementary File 1

Table S1. Discrimination parameters for predicting AKI and mortality according to access

Please find supplementary file(s);

http://dx.doi.org/10.1253/circj.CJ-14-1242 